\title{
Trends in Incidence and Factors Affecting Survival of Patients With Cholangiocarcinoma in the United States
}

\author{
Shiva Kumar R. Mukkamalla, MD, MPH ${ }^{a}$; Hussain M. Naseri, MDª Byung M. Kim, MDª \\ Steven C. Katz, $\mathrm{MD}^{\mathrm{b}}$; and Vincent A. Armenio, $\mathrm{MD}^{\mathrm{a}}$
}

\begin{abstract}
Background: Cholangiocarcinoma (CCA) includes cancers arising from the intrahepatic and extrahepatic bile ducts. The etiology and pathogenesis of CCA remain poorly understood. This is the first study investigating both incidence patterns of CCA from 1973 through 2012 and demographic, clinical, and treatment variables affecting survival of patients with CCA. Patients and Methods: Using the SEER database, age-adjusted incidence rates were evaluated from 1973-2012 using SEER*Stat software. A retrospective cohort of 26,994 patients diagnosed with CCA from 1973-2008 was identified for survival analysis. Cox proportional hazards models were used to perform multivariate survival analysis. Results: Overall incidence of CCA increased by 65\% from 1973-2012. Extrahepatic CCA (ECC) remained more common than intrahepatic CCA (ICC), whereas the incidence rates for ICC increased by $350 \%$ compared with a $20 \%$ increase seen with ECC. Men belonging to non-African American and non-Caucasian ethnicities had the highest incidence rates of CCA. This trend persisted throughout the study period, although African Americans and Caucasians saw 50\% and 59\% increases in incidence rates, respectively, compared with a $9 \%$ increase among other races. Median overall survival (OS) was 8 months in patients with ECC compared with 4 months in those with ICC. Our survival analysis found Hispanic women to have the best 5 -year survival outcome $(P<.0001)$. OS diminished with age $(P<.0001)$, and ECC had better survival outcomes compared with ICC $(P<.0001)$. Patients who were married, were nonsmokers, belonged to a higher income class, and underwent surgery had better survival outcomes compared with others $(P<.0001)$. Conclusions: This is the most up-to-date study of CCA from the SEER registry that shows temporal patterns of increasing incidence of CCA across different races, sexes, and ethnicities. We identified age, sex, race, marital status, income, smoking status, anatomic location of CCA, tumor grade, tumor stage, radiation, and surgery as independent prognostic factors for OS in patients with CCA.
\end{abstract}

Cholangiocarcinomas (CCAs) are rare cancers that arise from the intrahepatic or extrahepatic biliary ductal epithelium, which are classified into intrahepatic or extrahepatic CCAs (ICCs and ECCs, respectively). ECCs are further stratified based on anatomic location into perihilar and distal CCAs. CCA accounts for approximately $10 \%$ to $15 \%$ of primary hepatic malignancies. ${ }^{1}$ In the United States, approximately 5,000 new cases of CCA are diagnosed annually, with relatively equal distribution between ICC and ECC. ${ }^{2}$ Incidence and mor-

aDivision of Hematology and Oncology, and 'Division of Surgical Oncology, Roger Williams Medical Center/Boston University School of Medicine, Providence, Rhode Island.

Submitted July 8, 2017; accepted for publication November 8, 2017.

The authors have disclosed that they have no financial interests, arrangements, affiliations, or commercial interests with the manufacturers of any products discussed in this article or their competitors. tality of CCA have been reported to be increasing in the United States. ${ }^{3}$ Although reasons for the increased incidence are unclear, it is thought to be partly from improved diagnostic techniques with higher detection rates and the increased incidence of hepatitis $\mathrm{C}$ virus infection. ${ }^{4}$

The etiology and pathogenesis of CCA remain poorly understood. Although most CCAs can arise de novo, risk factors include primary sclerosing cholangitis (PSC), alcohol consumption, liver cirrhosis, and

Author contributions: Study concept: Mukkamalla, Naseri, Armenio. Study design: Mukkamalla, Katz, Armenio. Data acquisition: Mukkamalla. Data analysis: Mukkamalla. Literature review: Mukkamalla, Naseri, Kim. Manuscript preparation: Mukkamalla, Naseri, Kim. Directed, reviewed and provided expert supervision: Katz, Armenio.

Correspondence: Shiva Kumar R. Mukkamalla, MD, MPH, 30 Oaklawn

Avenue, Apartment 303, Cranston, RI 02920.

Email: shiva.mukkamalla@gmail.com 
chronic viral hepatitis infections. ${ }^{2,4-16}$ The prevalence of CCA increases steadily with age, peaking in the seventh decade. ${ }^{17}$ Although prevalence does not vary greatly by race or sex, it has been shown that in the United States, Hispanic men are at the highest risk for $\mathrm{CCA}^{18-20}$ and African American men have the lowest risk, whereas Asians/Pacific Islanders and Caucasians have prevalence rates ranging between these 2 groups. ${ }^{20,21} \mathrm{CCA}$ has a high male predominance, except for Hispanic women, who have higher rates of ICC compared with Hispanic men. ${ }^{20,22}$ Cumulative CCA mortality rates have increased by $39 \%$ because of increased incidence and mortality rates from ICC, which is reportedly highest in American Indians and Asians. ${ }^{23}$

This retrospective study examined the evolving epidemiologic trends in incidence of CCA in the United States, while analyzing the influence of various biographic, demographic, and clinical variables on the survival of patients with CCA.

\section{Patients and Methods}

\section{Data Source}

CCA-specific data were obtained from the SEER 18 registry, which included cases from 1973 through 2013. ${ }^{24}$ This registry includes 9.1 million patient records encompassing all cancers, representing approximately $28 \%$ of all newly diagnosed cancers in the United States. ${ }^{25}$ The SEER*Stat statistical software package was used to calculate incidence rates from 1973-2012, which were age-adjusted to the US standard population of 2000 . Incidence patterns were further examined by primary site of CCA (ICC or ECC), sex, and ethnicity. Survival analysis was performed using data from the SEER 18 registry database for 1973-2013, by including cases diagnosed from 1973 through 2008. A study endpoint of 2008 was chosen to allow for a minimum of 5 years followup, because the last data entry point for this cohort was in December 2013.

\section{Study Population}

CCA was defined according to the ICD-O-3 codes of C221 for ICC and C240-249 for ECC. We identified a total of 26,994 patients diagnosed with CCA from 1973-2008 after using age-mandated eligibility criteria of $\geq 18$ years. We excluded patients diagnosed with CCA based on clinical judgement alone, as well as those lacking documentation on ethnicity, survival duration, and smoking status, and those with overlapping lesions of biliary tract. Overlapping lesions were defined as tumors overlapping the boundaries of $\geq 2$ categories or subcategories, whose point of origin could not be determined. Gallbladder cancers and combined hepatocellular carcinomas/ CCAs were also excluded.

\section{Methods and Variables}

The primary endpoint of this study was 5-year overall survival (OS). In patients alive at last follow-up, OS was censored at 60 months. We chose to analyze age as an ordinal variable. Patients were categorized into 4 ethnic groups: Caucasian, African American, Hispanic, and other races, which included American Indian/Alaska Native, Asian, Fiji Islander, Guamanian, Hawaiian, Micronesian, Pacific Islander, Polynesian, and Samoan. Cases were classified into ICC and ECC based on reported primary site. Extrahepatic sites also included ampulla of Vater and biliary tract, not otherwise specified. CCA was divided according to the grade at presentation into well differentiated (grade I), moderately differentiated (grade II), poorly differentiated (grade III), undifferentiated/anaplastic (grade IV), or unknown. Based on surgery and radiation information in the SEER database, we redefined treatment types into unknown, no treatment, surgery only, radiation only, and surgery and radiation. Patients were divided into quartiles based on "percent of population that ever smoked" reported from their respective counties for 2000 to 2003. The first quartile represents the lowest smoking rate, whereas the fourth quartile represents the highest smoking rate. Similarly, patients were classified into quartiles based on the median family incomes of their respective counties from 2009 to 2013, with the first quartile having the lowest income level and the fourth quartile having the highest.

\section{Statistical Analysis}

SEER*Stat version 8.3.2 was used to calculate incidence rates, which were age-adjusted to the US standard population of 2000 . Incidence patterns were further stratified according to the primary tumor site, sex, and ethnicity. For survival analysis, patients were stratified by age; sex; ethnicity; primary site, grade, and stage of tumor; surgery; treatment type; current marital status; smoking status; and in- 
Mukkamalla et al

come level. Descriptive statistics were performed for categorical and ordinal variables using frequencies. Differences among subgroups were evaluated using the chi-square test. The primary outcome was 5-year OS. Cox proportional hazards model was used to perform multivariate analysis and was constructed using age as an ordinal variable. Sex; ethnicity; primary site, grade, and stage of tumor; treatment type; and marital status were treated as categorical variables, whereas smoking status and income level were treated as ordinal variables. Hazard ratios (HRs) and 95\% CIs were generated, with an $\mathrm{HR}<1.0$ indicating a survival benefit. All tests of statistical significance were conducted using $P<.05$ as the significance level. Statistical analyses related to survival were performed using SAS 9.4 (SAS Institute, Cary, NC).

\section{Results}

\section{Patient Characteristics}

Our selection criteria identified a total of 26,994 patients diagnosed with CCA from 1973-2008 after using age-mandated eligibility criteria of $\geq 18$ years and other exclusion criteria as outlined earlier. Within the study cohort, 7,089 had ICC (26.3\%) and 19,905 had ECC (73.7\%). Median age was 70 years for ICC and 72 years for ECC. Caucasians constituted the predominant racial group in the study cohort. No significant differences were seen in sex or marital status among ICC and ECC subgroups. Patients with ECC received higher rates of surgery with or without radiation compared with those with ICC; 70\% of patients with ICC did not receive any kind of radiation or surgical intervention, whereas this number was low at $48 \%$ among patients with ECC (Table 1). Unfortunately, staging information was not available in $>70 \%$ of ECC and ICC cases.

\section{Incidence Patterns}

The age-adjusted incidence rates of CCA increased by almost $65 \%$ from 1.7 per 100,000 in 1973 through 1975 to 2.8 per 100,000 in 2011 through 2012 (Figure 1). Using data from all available SEER registries for these periods, we noted a continued increase in age-adjusted incidence rates of both ICC and ECC (Figure 2). Age-adjusted incidence rates of ICC increased by $350 \%$ : from 0.2 per 100,000 in $1973-$ 1975 to 0.9 per 100,000 in 2011-2012, whereas incidence rates of ECC increased by approximately

\begin{tabular}{|c|c|c|c|}
\hline Characteristics & $\begin{array}{c}\text { ICC } \\
(\mathrm{N}=7,089 ; 26.26 \%)\end{array}$ & $\begin{array}{c}\text { ECC } \\
(\mathrm{N}=19,905 ; 73.74 \%)\end{array}$ & $P$ Value \\
\hline Median age, y & 70 & 72 & - \\
\hline Race, n (\%) & & & $<.0001$ \\
\hline Caucasian & $4,932(69.57)$ & $14,271(71.7)$ & \\
\hline African American & $494(6.97)$ & $1,377(6.92)$ & \\
\hline Hispanic & $732(10.33)$ & $2,072(10.41)$ & \\
\hline Other $^{\mathrm{a}}$ & $931(13.13)$ & $2,185(10.98)$ & \\
\hline Sex, n (\%) & & & .48 \\
\hline Female & $3,432(48.41)$ & $9,540(47.93)$ & \\
\hline Male & $3,657(51.59)$ & $10,365(52.07)$ & \\
\hline Marital status, n (\%) & & & .87 \\
\hline Married & $3,994(56.34)$ & $11,236(56.45)$ & \\
\hline Not married & $3,095(43.66)$ & $8,669(43.55)$ & \\
\hline Year of diagnosis, n (\%) & & & $<.0001$ \\
\hline $1973-1980$ & $239(3.37)$ & $1,893(9.51)$ & \\
\hline $1981-1990$ & $643(9.07)$ & $2,741(13.77)$ & \\
\hline $1991-2000$ & $2,718(38.34)$ & $4,915(24.69)$ & \\
\hline $2001-2008$ & $3,489(49.22)$ & $10,356(52.03)$ & \\
\hline Income, quartile, n (\%) & & & $<.0001$ \\
\hline 1st, low & $1,556(21.95)$ & $5,209(26.17)$ & \\
\hline 2nd & $1,873(26.42)$ & $5,021(25.22)$ & \\
\hline $3 r d$ & $1,684(23.76)$ & $4,997(25.1)$ & \\
\hline 4th, high & $1,976(27.87)$ & $4,678(23.5)$ & \\
\hline Smoking, quartile, n (\%) & & & $<.0001$ \\
\hline 1st, low & $1,864(26.29)$ & $4,568(22.95)$ & \\
\hline 2nd & $1,982(27.96)$ & $5,393(27.09)$ & \\
\hline $3 r d$ & $1,612(22.74)$ & $4,871(24.47)$ & \\
\hline 4th, high & $1,631(23.01)$ & $5,073(25.49)$ & \\
\hline Grade, n (\%) & & & $<.0001$ \\
\hline Well differentiated, I & $367(5.18)$ & $2,120(10.65)$ & \\
\hline $\begin{array}{l}\text { Moderately } \\
\text { differentiated, II }\end{array}$ & $912(12.87)$ & $4,762(23.92)$ & \\
\hline $\begin{array}{l}\text { Poorly } \\
\text { differentiated, III }\end{array}$ & $901(12.71)$ & $3,392(17.04)$ & \\
\hline Undifferentiated, IV & $67(0.95)$ & $235(1.18)$ & \\
\hline Unknown & $4,842(68.3)$ & $9,396(47.2)$ & \\
\hline Stage, n (\%) & & & $<.0001$ \\
\hline 1 & $417(5.88)$ & $1,484(7.46)$ & \\
\hline II & $121(1.71)$ & $1,525(7.66)$ & \\
\hline III & $512(7.22)$ & $782(3.93)$ & \\
\hline IV & $714(10.07)$ & $1,582(7.95)$ & \\
\hline Unknown & $5,325(75.12)$ & $14,532(73.01)$ & \\
\hline Treatment, n (\%) & & & $<.0001$ \\
\hline Radiation & $868(12.24)$ & $1,588(7.98)$ & \\
\hline Surgery & $788(11.12)$ & $5,924(29.76)$ & \\
\hline Radiation + surgery & $247(3.48)$ & $1,876(9.42)$ & \\
\hline None & $4,972(70.14)$ & $9,641(48.44)$ & \\
\hline Unknown & $214(3.02)$ & $876(4.4)$ & \\
\hline
\end{tabular}

Abbreviations: ECC, extrahepatic cholangiocarcinoma; ICC, intrahepatic cholangiocarcinoma.

ancludes American Indian/Alaska Native, Asian, Fiji Islander, Guamanian, Hawaiian, Micronesian, Pacific Islander, Polynesian, and Samoan. 
Trends and Survival in Cholangiocarcinoma

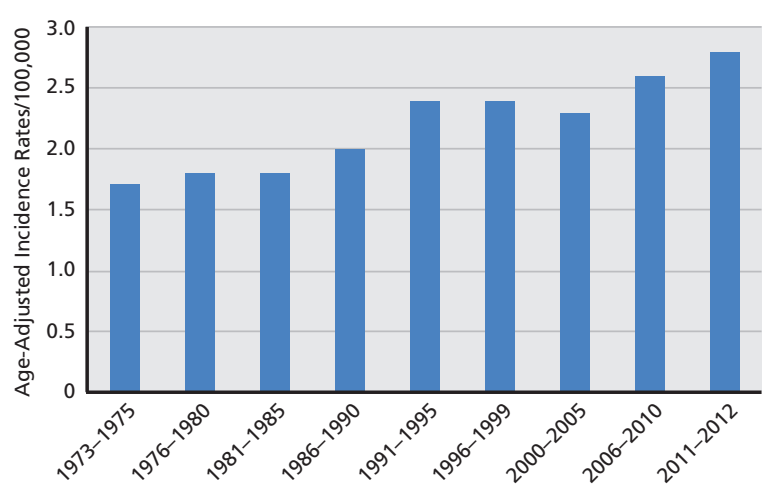

Figure 1. Temporal trends in age-adjusted incidence rates of cholangiocarcinoma from 1973-2012.

20\%: from 1.6 per 100,000 in $1973-1975$ to 1.9 per 100,000 in 2011-2012. In general, the incidence of ECC was higher than ICC across all time intervals. Sex-based disparities in incidence patterns of CCA were clearly depicted across all time intervals (Figure 3). Age-adjusted incidence rates from $1973-1975$ to $2011-2012$ increased by $65 \%$ in men: from 2 per 100,000 to 3.3 per 100,000 , respectively, whereas in women the incidence rates increased by $53 \%$ : from 1.5 per 100,000 to 2.3 per 100,000 . Among different racial groups, African Americans had the lowest age-adjusted incidence rates followed by Caucasians, who had slightly higher rates, whereas the incidence rate among other races was much higher (Figure 4). All of the groups demonstrated a progressive increase in age-adjusted incidence rates between 1973-1975 and 2011-2012, with Caucasians showing a 59\% increase, African

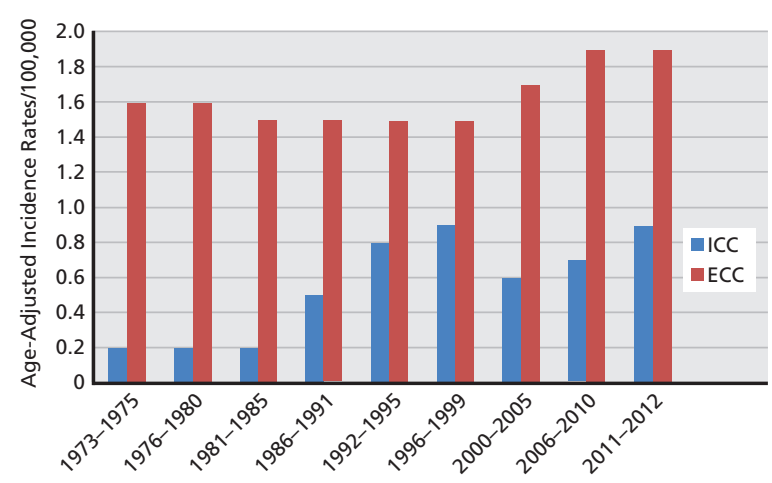

Figure 2. Temporal trends in age-adjusted incidence rates of cholangiocarcinoma by anatomic location.

Abbreviations: ECC, extrahepatic cholangiocarcinoma; ICC, intrahepatic cholangiocarcinoma.

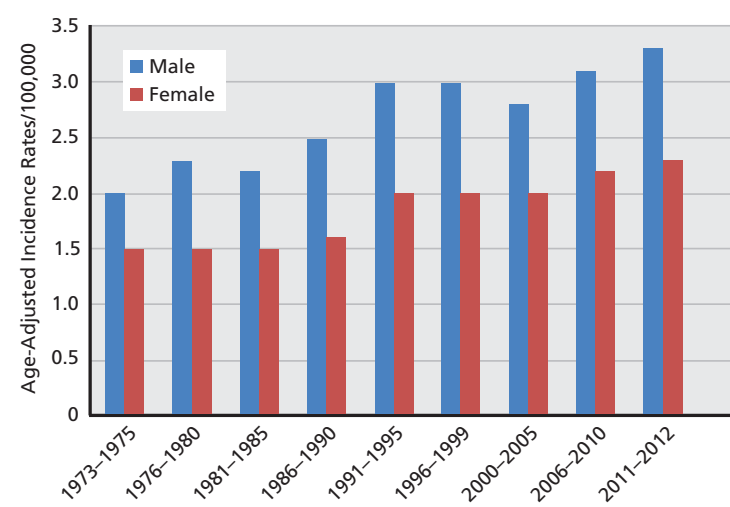

Figure 3. Temporal trends in age-adjusted incidence rates of cholangiocarcinoma by sex.

Americans a $50 \%$ increase, and other races a $9 \%$ increase.

\section{Survival Analysis}

Median OS in all patients with CCA was 7 months, whereas it was 8 months in ECC and 4 months in ICC. The prognostic significance of several social, clinical, and tumor characteristics is shown in Table 2. In multivariate analysis, age, sex, race, marital status, year of diagnosis, income, smoking, primary tumor site, tumor grade, tumor stage, and treatment type were significantly associated with OS. Women had a significantly lower mortality risk compared with men, and African Americans had the highest mortality risk among the various racial groups studied. Although the 5-year mortality rates were low for the African American population, the adjusted HR indicate that Hispanics had the best survival outcome from CCA.

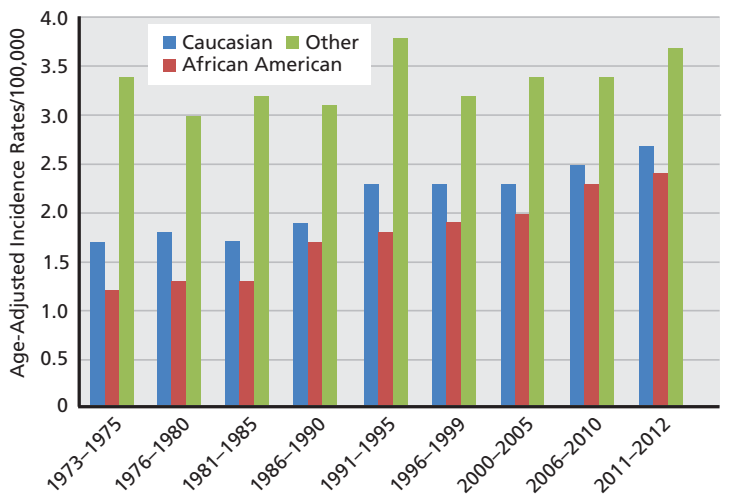

Figure 4. Temporal trends in age-adjusted incidence rates of cholangiocarcinoma among different ethnic groups. 
Mukkamalla et al

\begin{tabular}{|c|c|c|c|c|}
\hline Characteristics & $\begin{array}{c}\text { 5-Year } \\
\text { Mortality } \\
\text { Rate, \% }\end{array}$ & Hazard Ratio & $95 \% \mathrm{Cl}$ & $P$ Value \\
\hline Age, $y$ & & & & $<.0001$ \\
\hline $18-34$ & 69.9 & Ref $=1$ & & \\
\hline $35-49$ & 77.86 & 1.276 & $1.075-1.515$ & \\
\hline $50-64$ & 83.02 & 1.436 & $1.216-1.695$ & \\
\hline$\geq 65$ & 91.41 & 1.877 & $1.592-2.213$ & \\
\hline Race & & & & $<.0001$ \\
\hline Caucasian & 89.36 & Ref $=1$ & & \\
\hline African American & 83.99 & 1.081 & $1.027-1.138$ & \\
\hline Hispanic & 85.46 & 0.896 & $0.856-0.938$ & \\
\hline Othera & 89.32 & 0.903 & $0.865-0.942$ & \\
\hline Sex & & & & $<.0001$ \\
\hline Female & 88.6 & Ref $=1$ & & \\
\hline Male & 88.08 & 1.065 & $1.037-1.094$ & \\
\hline Marital status & & & & $<.0001$ \\
\hline Married & 86.64 & Ref $=1$ & & \\
\hline Not married & 90.5 & 1.090 & $1.060-1.120$ & \\
\hline \multicolumn{5}{|l|}{ Year of diagnosis } \\
\hline $1973-1980$ & 92.17 & Ref $=1$ & & \\
\hline 1981-1990 & 89.86 & 0.888 & $0.839-0.940$ & \\
\hline $1991-2000$ & 88.56 & 0.752 & $0.713-0.792$ & \\
\hline $2001-2008$ & 87.23 & 0.744 & $0.706-0.784$ & \\
\hline Income, quartile & & & & $<.0001$ \\
\hline $1 s t$ low & 89.74 & Ref $=1$ & & \\
\hline 2nd & 87.83 & 0.936 & $0.899-0.975$ & \\
\hline 3 rd & 87.95 & 0.901 & $0.868-0.936$ & \\
\hline 4th, high & 87.75 & 0.856 & $0.821-0.892$ & \\
\hline Smoking, quartile & & & & $<.0001$ \\
\hline 1st, low & 86.55 & Ref $=1$ & & \\
\hline 2nd & 89.13 & 1.070 & $1.026-1.115$ & \\
\hline $3 r d$ & 87.69 & 1.030 & $0.987-1.074$ & \\
\hline 4th, high & 89.77 & 1.002 & $0.959-1.047$ & \\
\hline Site & & & & $<.0001$ \\
\hline $\mathrm{ICC}$ & 94.05 & $\operatorname{Ref}=1$ & & \\
\hline ECC & 86.29 & 0.856 & $0.831-0.883$ & \\
\hline Grade & & & & $<.0001$ \\
\hline $\begin{array}{l}\text { Well } \\
\text { differentiated, I }\end{array}$ & 75.47 & Ref $=1$ & & \\
\hline $\begin{array}{l}\text { Moderately } \\
\text { differentiated, II }\end{array}$ & 79.91 & 1.287 & $1.219-1.359$ & \\
\hline $\begin{array}{l}\text { Poorly differentiated, } \\
\text { III }\end{array}$ & 88.26 & 1.634 & $1.545-1.728$ & \\
\hline Undifferentiated, IV & 92.72 & 1.773 & $1.563-2.011$ & \\
\hline Unknown & 93.85 & 1.358 & $1.292-1.429$ & \\
\hline Stage & & & & $<.0001$ \\
\hline 1 & 74.17 & Ref $=1$ & & \\
\hline ॥ & 79.47 & 1.284 & $1.190-1.385$ & \\
\hline III & 87.94 & 1.365 & $1.262-1.477$ & \\
\hline IV & 97.13 & 1.787 & $1.670-1.914$ & \\
\hline Unknown & 89.42 & 1.4 & $1.321-1.484$ & \\
\hline Treatment & & & & $<.0001$ \\
\hline None & 96.76 & Ref $=1$ & & \\
\hline Radiation & 96.42 & 0.693 & $0.663-0.724$ & \\
\hline Surgery & 70.28 & 0.342 & $0.329-0.355$ & \\
\hline Surgery + radiation & 75.69 & 0.360 & $0.340-0.381$ & \\
\hline Unknown & 92.75 & 0.698 & $0.654-0.745$ & \\
\hline
\end{tabular}

Abbreviations: ECC, extrahepatic cholangiocarcinoma; ICC, intrahepatic cholangiocarcinoma.

alncludes American Indian/Alaska Native, Asian, Fiji Islander, Guamanian, Hawaiian, Micronesian, Pacific Islander, Polynesian, and Samoan.
Marital status also had significant effect on OS, as did income level and smoking status. Improved survival was demonstrated in patients with later years of diagnosis. On multivariate analysis, the HR for mortality of patients diagnosed in 2001-2008 was 0.744 (95\% CI, 0.706-0.784) compared with 1973-1980. Tumor character had a significant impact on survival; as expected, patients with poorly differentiated tumors had worse survival than those with moderately or welldifferentiated tumors (Table 2). We also found that ECC was associated with a survival benefit compared with ICC (HR, 0.856; 95\% CI, 0.831-0.883). Surgery alone (HR, 0.342; 95\% CI, 0.329-0.355) or surgery with radiation (HR, 0.360; 95\% CI, 0.340-0.381) were associated with significantly better survival outcomes compared with radiation alone (HR, 0.693; 95\% CI, 0.663-0.724) or no treatment.

\section{Discussion}

This study cohort of patients with CCA from the SEER database demonstrates a persistent increase in the incidence of CCA in the United States from 1973-2012. It confirms the findings reported in earlier studies, ${ }^{4,23,26}$ while expanding the data analyzed to include cases diagnosed up to 2012. Although the highest incidence rates were found in men from nonCaucasian and non-African American ethnic backgrounds, the increase in incidence affected men and women of all racial groups.

The reasons for increased CCA incidence are not clearly delineated. If it is attributed to increasing incidence of PSC, ${ }^{13-16}$ there should have been a shift toward increasing diagnosis of CCA at a younger age and more frequently in women. Our study, similar to a prior study by Shaib et al, ${ }^{4}$ showed that the increasing incidence of CCA was not associated with a shift toward younger age or relatively increased female predisposition. Shaib et $\mathrm{al}^{4}$ also examined the increasing incidence of ICC in the United States and suggested a true increase, which could not be completely explained by improved detection alone. Incidence of CCA is known to be exceptionally high in certain parts of the world, including Chile, Bolivia, South Korea, and North Thailand. ${ }^{27}$ Additionally, other risk factors for CCA, such as parasitic infections (eg, Clonorchis sinensis), could have affected the immigrant population from these regions (mostly from Southeast Asia). We were able to confirm 
Trends and Survival in Cholangiocarcinoma

the higher rates of CCA among American Indian/ Alaska Natives, Hispanics, and Asians/Pacific Islanders (represented in the ethnic subgroup "others") compared with African Americans and Caucasians. ${ }^{26}$

Hepatitis $B$ and $C$ virus infections and liver cirrhosis have all been suggested as potential risk factors for CCA, 2,4-12 with 3 studies from Asia ${ }^{28,29}$ and Italy ${ }^{6}$ showing these to be associated with increased incidence rates of CCA. However, there have been no studies on CCA and viral infections conducted in the United States, thereby limiting the generalizability of these results to the US population. We could also attribute the increased CCA incidence to ever-improving and more specialized diagnostic modalities developed over the years, but there is a paucity of evidence to support this.

For survival analysis, we used a cohort consisting of 26,994 patients diagnosed with CCA from 1973-2008. As expected, we found tumor characteristics such as primary site, grade, and stage to be associated with survival in CCA. Notably, there was a survival advantage attributable to ECC compared with ICC (HR, 0.856; 95\% CI, 0.831-0.883). Survival differences between ICC and ECC may reflect differences in pathogenesis and tumor biology. This is in contrast to the findings of ABC-02 trial, which showed better outcomes for patients with ICC. ${ }^{30}$ However, SEER represents population cancer registries that consist of unselected patient populations with a great variation in treatments, compared with clinical trials that contain selected populations receiving predefined treatment. Furthermore, ECC is now classified into perihilar and distal ECC, which are managed differently ${ }^{31}$; perihilar and distal ECC were included under a single entity of ECC until the AJCC 7th edition. This study analyzed perihilar and distal ECC as a single entity.

In addition to tumor characteristics, patient features, such as age and sex, also had significant prognostic impact. Ethnicity, which is often described in the literature as having a prominent prognostic role, did have a significant effect on survival. We found Hispanics had a better survival outcome compared with other ethnicities, whereas non-Hispanic blacks had the worst survival outcome. Survival for our youngest age group (18-34 years) was almost 2 -fold higher than for the oldest age group ( $\geq 65$ years); this could be representative of higher rates of treatment in younger patients and is unlikely due to any dif- ferences in tumor biology in the older patients. We could not address the role of systemic chemotherapy on OS in the current study due to lack of information in SEER. Consistent with previous reports, we found women had better survival outcomes than men. However, no definitive molecular pathways have been identified to explain this association.

Other demographic variables, including marital status, income levels, and smoking status, were associated with prognostic implications. Individuals who were married had better survival outcomes than those who were not married (HR, 1.09; 95\% CI, 1.0601.120), and people with higher income levels did better than those with low income (HR, 0.856; 95\% CI, 0.821-0.892). These findings are attributable to the availability of good social and economic support. As expected, smoking did worsen the probability of survival.

Multivariate analysis revealed a continued reduction in mortality from 1973-2008, attributable to early diagnosis and improved surgical techniques, along with increased use of chemotherapy and radiation over time. We noted surgery alone or surgery with radiation had the best outcomes in terms of OS. Improvements in the safety of hepatic resection $^{32}$ and pancreaticoduodenectomy ${ }^{33}$ may have resulted in a higher proportion of margin-negative resections, resulting in better oncologic results. Based on our findings, radiation alone did not confer any improvement in OS compared with surgery alone or with radiation, although it showed better results than no treatment. Outcomes for patients undergoing surgery with or without radiation improved over the decades, whereas no improvement was seen with radiation alone (Table 3 ).

This study has the limitations of being retrospective in nature. The impact of systemic chemotherapy on survival of CCA could not be ascertained in this multivariate analysis due to the lack of chemother-

\begin{tabular}{|lcccc|}
\hline \multirow{4}{*}{$\begin{array}{c}\text { Table 3. 5-Year Survival Outcomes by Year of } \\
\text { Diagnosis and Treatment }(\boldsymbol{P}<\text {.0001) }\end{array}$} \\
\cline { 2 - 5 } Year of Diagnosis & RT & Surgery & Surgery + RT & No Treatment \\
\hline $1973-1980$ & 95.21 & 80.47 & 88.89 & 97.8 \\
\hline $1981-1990$ & 95.37 & 73.55 & 85.65 & 98.49 \\
\hline $1991-2000$ & 97.2 & 70.44 & 78.98 & 96.48 \\
\hline $2001-2008$ & 96.43 & 67.19 & 71.48 & 96.46 \\
\hline
\end{tabular}

Abbreviation: $\mathrm{RT}$, radiotherapy. 
Mukkamalla et al

apy-specific information in the SEER database, such as combination regimen, dose, and cycles.

\section{Conclusions}

This study identified various biographic, demographic, and clinical variables that have a significant prognostic influence on OS in patients with CCA. This is the most up-to-date population-based analysis that demonstrates a continued increase in CCA incidence in the United States. The factors causing this increase are not very clear and require large controlled studies to examine the direct role of potential underlying risk factors.

\section{References}

1. Altekruse SF, Devesa SS, Dickie LA, et al. Histological classification of liver and intrahepatic bile duct cancers in SEER registries. J Registry Manag 2011;38:201-205.

2. Shaib YH, El-Serag HB, Nooka AK, et al. Risk factors for intrahepatic and extrahepatic cholangiocarcinoma: a hospital-based case-control study. Am J Gastroenterol 2007;102:1016-1021.

3. Saha SK, Zhu AX, Fuchs CS, et al. Forty-year trends in cholangiocarcinoma incidence in the U.S.: intrahepatic disease on the rise. Oncologist 2016;21:594-599.

4. Shaib YH, Davila JA, McGlynn K, El-Serag HB. Rising incidence of intrahepatic cholangiocarcinoma in the United States: a true increase? J Hepatol 2004;40:472-477.

5. Welzel TM, Mellemkjaer L, Gloria G, et al. Risk factors for intrahepatic cholangiocarcinoma in a low-risk population: a nationwide case-control study. Int J Cancer 2007;120:638-641.

6. Donato F, Gelatti U, Tagger A, et al. Intrahepatic cholangiocarcinoma and hepatitis $\mathrm{C}$ and $\mathrm{B}$ virus infection, alcohol intake, and hepatolithiasis: a case-control study in Italy. Cancer Causes Control 2001;12:959-964.

7. El-Serag HB, Engels EA, Landgren O, et al. Risk of hepatobiliary and pancreatic cancers after hepatitis $\mathrm{C}$ virus infection: a population-based study of U.S. veterans. Hepatology 2009;49:116-123.

8. Shaib YH, El-Serag HB, Davila JA, et al. Risk factors of intrahepatic cholangiocarcinoma in the United States: a case-control study. Gastroenterology 2005;128:620-626.

9. Lee TY, Lee SS, Jung SW, et al. Hepatitis B virus infection and intrahepatic cholangiocarcinoma in Korea: a case-control study. Am J Gastroenterol 2008;103:1716-1720.

10. Zhou YM, Yin ZF, Yang JM, et al. Risk factors for intrahepatic cholangiocarcinoma: a case-control study in China. World J Gastroenterol 2008;14:632-635.

11. Sekiya S, Suzuki A. Intrahepatic cholangiocarcinoma can arise from notchmediated conversion of hepatocytes. J Clin Invest 2012;122:3914-3918.

12. Yamamoto $S$, Kubo $S$, Hai $S$, et al. Hepatitis $C$ virus infection as a likely etiology of intrahepatic cholangiocarcinoma. Cancer Sci 2004;95:592 595.

13. Chapman MH, Webster GJ, Bannoo S, et al. Cholangiocarcinoma and dominant strictures in patients with primary sclerosing cholangitis: a 25 year single-centre experience. Eur J Gastroenterol Hepatol 2012;24:10511058.

14. Bergquist A, Ekbom A, Olsson R, et al. Hepatic and extrahepatic malignancies in primary sclerosing cholangitis. J Hepatol 2002;36:321327.

15. Chapman R, Fevery J, Kalloo A, et al. Diagnosis and management of primary sclerosing cholangitis. Hepatology 2010;51:660-678.
16. Claessen MM, Vleggaar FP, Tytgat KM, et al. High lifetime risk of cancer in primary sclerosing cholangitis. J Hepatol 2009;50:158-164.

17. Khan SA, Toledano MB, Taylor-Robinson SD. Epidemiology, risk factors, and pathogenesis of cholangiocarcinoma. HPB (Oxford) 2008;10:77-82.

18. Tyson GL, El-Serag HB. Risk factors for cholangiocarcinoma. Hepatology 2011;54:173-184.

19. Shaib Y, El-Serag HB. The epidemiology of cholangiocarcinoma. Semin Liver Dis 2004;24:115-125.

20. Everhart JE, Ruhl CE. Burden of digestive diseases in the United States part III: liver, biliary tract, and pancreas. Gastroenterology 2009;136:1134 1144 .

21. Blechacz BR, Gores GJ. Cholangiocarcinoma. Clin Liver Dis 2008;12:131150.

22. McGlynn KA, Tarone RE, El-Serag HB. A comparison of trends in the incidence of hepatocellular carcinoma and intrahepatic cholangiocarcinoma in the United States. Cancer Epidemiol Biomarkers Prev 2006;15:1198-1203.

23. Razumilava N, Gores GJ. Cholangiocarcinoma. Lancet 2014;383:21682179.

24. SEER*Stat Databases: November 2014 Submission. Incidence - SEER 18 Regs Research Data + Hurricane Katrina Impacted Louisiana Cases, Nov 2014 Sub (1973-2012 varying). SEER Web site. Available at: https:/ seer.cancer.gov/data-software/documentation/seerstat/nov2014/. Accessed March 23, 2018.

25. Overview of the SEER Program. SEER Web site. Available at: https://seer. cancer.gov/about/overview.html. Accessed November 6, 2017.

26. Castro FA, Koshiol J, Hsing AW, Devesa SS. Biliary tract cancer incidence in the United States-demographic and temporal variations by anatomic site. Int J Cancer 2013;133:1664-1671.

27. Fitzmaurice C, Dicker D, Pain A, et al. The global burden of cancer 2013. JAMA Oncol 2015;1:505-527.

28. Shin HR, Lee CU, Park HJ, et al. Hepatitis B and C virus, clonorchis sinensis for the risk of liver cancer: a case-control study in Pusan, Korea. Int J Epidemiol 1996;25:933-940.

29. Kobayashi M, Ikeda $K$, Saitoh $S$, et al. Incidence of primary cholangiocellular carcinoma of the liver in Japanese patients with hepatiti C virus-related cirrhosis. Cancer 2000;88:2471-2477.

30. Valle J, Wasan H, Palmer DH, et al. Cisplatin plus gemcitabine versus gemcitabine for biliary tract cancer. N Engl J Med 2010;362:1273-1281.

31. Blechacz B. Cholangiocarcinoma: current knowledge and new developments. Gut Liver 2017;11:13-26.

32. Kooby DA, Jarnagin WR. Surgical management of hepatic malignancy. Cancer Invest 2004:22:283-303.

33. Cameron JL, Riall TS, Coleman J, Belcher KA. One thousand consecutive pancreaticoduodenectomies. Ann Surg 2006;244:10-15. 